EPJ Web of Conferences 106, 02016 (2016)

DOI: $10.1051 /$ epjconf/201610602016

(C) Owned by the authors, published by EDP Sciences, 2016

\title{
VVER Knowledge Preservation and Transfer within the Frame of CORONA Project Activities
}

\author{
Mladen Mitev ${ }^{1}$, Enrico Corniani ${ }^{2}$, Maria Manolova $^{1}$, Lybomir Pironkov $^{3}$, and Iskren Tsvetkov ${ }^{3}$ \\ ${ }^{1}$ INRNE-BAS, 72 Tzarigradsko shose, 1784 Sofia, Bulgaria \\ 2 JRC-IET, NL-1755 ZG Petten, The Netherlands \\ ${ }^{3}$ Kozloduy NPP Plc., 3321 Kozloduy, Bulgaria
}

\begin{abstract}
The CORONA project is funded by the European Commission under the FP7 programme with overall objective to establish a Regional Centre of Competence for VVER Technology and Nuclear Applications. The Centre will provide support and services for preservation and transfer of VVER-related nuclear knowledge as well as know-how and capacity building. Specific training schemes aimed at nuclear professionals and researchers, non-nuclear professionals and students are developed and implemented in cooperation with local, national and international training and educational institutions. Pilot trainings are executed for each specific target group to assess the applicability of the training materials. The training scheme implemented for nuclear professionals and researchers is focussed on the NPP Lifetime Management. The available knowledge on enhancing safety and performance of nuclear installations with VVER technology is used in the preparation of the training materials. The Online Multimedia Training Course on VVER Reactor Pressure Vessel Embrittlement and Integrity Assessment, developed by the joint effort of JRC-IET and IAEA is used in the training. The outcome collected from the trainees showed that the tool meets its primary goal of consolidating the existing knowledge on the VVER RPV Embrittlement and Integrity Assessment, provides adequate ground for transfer of this knowledge.
\end{abstract}

\section{Introduction}

The project CORONA "Establishment of Regional Centre of Competence for VVER Technology and Nuclear Applications" is co-funded by the European Commission under the FP7 programme with overall objective to establish a Regional Centre of Competence for VVER Technology and Nuclear Applications. It was started in December 2011. The project is 3 years long and 11 companies from 7 member states and associated countries are participating. The Regional Centre of Competence (RCC) will provide support and services for preservation and transfer of VVER-related nuclear knowledge as well as know-how and capacity building. Such an approach will ensure that existing VVER related training schemes will meet the International Atomic Energy Agency (IAEA) standards and generally recognized criteria in the European Union (EU).

This is an Open Access article distributed under the terms of the Creative Commons Attribution License 2.0, which permits unrestricted use, distribution, and reproduction in any medium, provided the original work is properly cited. 
The project structure is based on three general pillars:

- Training schemes for different target groups;

- VVER related knowledge management system, which will accumulate the available information;

- Specialized regional training centre for supporting VVER customers with theoretical and practical training sessions, training materials and general and special assignment training tools and facilities.

The expected outcome of the project is to develop new skills and competences within the context of the Nuclear Renaissance for implementing the EC decisions following SNETP NET [1] and SRA [2] and to contribute to the improvement of the safety and the reliability of the nuclear installations existing in EU. In long term vision, the project outcomes will serve as a base for evaluation of the options for establishment of the RCC as a legal entity and its joining to the European Nuclear Education Network (ENEN).

\section{Training Schemes Development}

The training scheme defines training programs, set of courses, training materials, training aids and various forms of training activities, designed to meet the requirements for the necessary professional knowledge and skills. The training schemes should provide certain knowledge, methods and forms in a way that ensures their good understanding and will help attract the trainees to nuclear activities.

The collection and analyses of the available VVER technology related knowledge was the base in the training schemes development process. The analyses shown that different target groups can benefit from the available knowledge. The specific training needs were identified and list of necessary skills and competencies for each of the following target groups was produced:

- Non-nuclear professionals and subcontractors;

- Students studying nuclear disciplines;

- Nuclear professionals and researchers.

The competences on "soft" skills for all the target groups were also identified. The concept of an accreditation/certification structure referring to ECVET (European Credit System for Vocational Education and Training) was defined. The description of general framework for all training schemes was prepared.

The training scheme for non-nuclear professionals and subcontractors included general education on the radiation detection and radiation protection principles, general knowledge on Nuclear Power Plant (NPP) electricity generation, VVER construction specifics and safety culture principles.

The training scheme developed for students studying nuclear disciplines brought additional in-depth knowledge on VVER reactor physics and safety, plant operation and life management.

The training scheme for nuclear professionals and researchers used as a base the whole VVER technology related knowledge for providing advanced skills and competences in operating and supporting a VVER based NPP. The available knowledge on enhancing safety and performance of nuclear installations with VVER technology was used in the preparation of the training materials. NPP Lifetime Management main activities/stages were described within their technical, economical, legal and safety culture aspect.

The training schemes were developed according to the Systematic Approach to Training (SAT) under the following five phases: Analysis, Design, Development, Implementation and Evaluation [3].

\section{Training Implementation and Evaluation}

Five pilot training courses were arranged within the project with the aim to evaluate the applicability of the elaborated training materials in their educational aspect. The participants were selected based 


\section{$15^{\text {th }}$ ISRD}

on their current level of education, expertise and professional achievements to assure the biggest possible coverage of all training program's aspects. This verification approach allows the refinement and improvement of the training materials based on the analyses of the feedback received from the trainees and observers.

\subsection{Pilot Training for Non-nuclear Professionals and Subcontractors}

The pilot training was developed as a test of on-the-job training experience of the Training Programme on advanced VVER technology specifics for non-nuclear professionals and subcontractors. The participants were trained to perform works related to VVER based NPP, radiation monitoring and radiation protection of places of ionizing radiation like NPP, medicine and industry applications, waste management, custom officers, dose control. The pilot training aims also to give the necessary competencies corresponding to the European Qualifications Framework (EQF) Level 3 to professionals working in support of nuclear applications as civil engineers, physical protection employees, government employees, secondary school teachers, journalists, etc. The training objective was to provide knowledge needed to start working in the nuclear related area with sufficient general nuclear knowledge and culture. The analysis of the pilot training results based on the replies of the test and interview of the trainees shown that the used approach is appropriate and could be disseminated among the interested parties. The interest showed from different professionals is an important indicator about the necessity to provide such kind of courses for non-nuclear professionals.

\subsection{Pilot Training for Students Studying Nuclear Disciplines}

The purpose of this training course is using different forms of training, like theoretical training, practical training, on-the-job training and simulator training, to enhance and overbuild the knowledge obtained in educational institutions through transfer of specialized knowledge. One such example is that the course reviewed the specific safety aspects of VVER technologies. During the course evaluation the trainees were very active and gave important recommendations on the improvement of the quality and accessibility of the training materials.

\subsection{Pilot Training for Nuclear Professionals and Researchers}

The most knowledge demanding training scheme is focused on the training for nuclear professionals and researchers. The training program should address the current and also the future needs of the enterprises and organizations in the nuclear fleet, to enhance management and professionals skills and to obtain additional professional competences of advanced technologies in nuclear.

Three pilot training were organized to cover the program extent:

- One week cross-cutting training on reactor physics, based on real reactor experiments performed on the LR-0 research reactor;

- Training on safety culture and soft skills, that covered topics related to Organizational Culture and Safety Culture; Human Performance Tools; Soft Skills Competences and Leadership Competences;

- Training in different aspects required for work in safe nuclear environment with sufficient general knowledge to participate in the activities related to nuclear facilities' lifetime management and extension.

The available knowledge on enhancing safety and performance of nuclear installations with VVER technology is used in the preparation of the training materials for the last course. NPP Lifetime Management main activities/stages were described within their technical, economical, legal and safety 
culture aspect. The course was evaluated by both trainees and observers. Important recommendations were made, but the overall opinion was that both the trainers and the training materials provide the most up to date knowledge on VVER NPP Lifetime Management aspects.

\section{Knowledge Management Approach}

To address the existing mobility challenge imposed by the spread of the state-of-the-art knowledge and expertise across the world, an approach to built online knowledge management system that contains the existing information on the VVER technology is utilized.

\subsection{Knowledge Management Portal for VVER Technology}

The framework of Knowledge Management Portal that will contain the available VVER technology related knowledge is established.

The identified objects of the Portal are to:

- Provide a structured system for easy, computer based access to any VVER related knowledge and information, which can be used by staff to maintain and improve their productivity and performance;

- Preserve VVER specific nuclear knowledge, skills and competencies;

- Facilitate communication for the sharing and growth of corporate knowledge.

Upon completion the portal will provide information on VVER Technology relevant to the key stakeholders from the nuclear power industry, scientific organizations, academia, industry and government.

IAEA declared interest in the implementation of CORONA project and to contribute to some of the activities, in particular the development of the Knowledge Management Portal.

\subsection{VVER RPV Training Tool}

Important part of the NPP Lifetime Management course was the full scale implementation of the Online Multimedia Training Course on VVER Reactor Pressure Vessel Embrittlement and Integrity Assessment [4]. The tool is developed by the joint effort of JRC-IET and IAEA and is available online on JRC webpage. The input of some of the most recognised experts in the VVER RPV Embrittlement was used. The tool consists of ten modular courses that cover the RPV Design Features, RPV Surveillance Programmes, Material and Environmental Factors, Irradiation Shift Prediction, etc. and a separate module on Integrity Assessment.

The incorporation of the tool in the pilot training has the goal to provide knowledge on VVER RPV embrittlement, but also the opportunity to receive feedback on the tool from experienced nuclear professionals and researchers was used. For the purpose each participant in the CORONA pilot training course was assigned with three topics to study. The topics were selected in a way to match the trainee background. The learning module was followed by an on-line multiple-choice format test where the student could assess his/her progress on the topic. All participants scored above $90 \%$ of correct answers, which shows the effectiveness of the Multimedia Training Course. The follow-up on the performed training was carried out by interviewing the participating researchers and nuclear professionals. The outcome of the interview showed that the tool meets its primary goal of consolidating the existing knowledge on the VVER RPV embrittlement and integrity assessment, provides adequate ground for transfer of this knowledge over the generational gap appeared during the last years and it is an important part of the training materials included in the training scheme for the nuclear professionals and researchers, developed within the framework of the CORONA Project. 


\section{$15^{\text {th }}$ ISRD}

\section{Conclusion}

Within the CORONA project activities the available knowledge on VVER related technology is collected, and new knowledge is developed to provide support and services for preservation and transfer of VVER related nuclear knowledge as well as know-how and capacity building. The information is made available electronically and will be contained within online Knowledge Management Portal. The online Multimedia Training Course on VVER Reactor Pressure Vessel Embrittlement and Integrity Assessment was successfully implemented in the training process and showed its viability as training option that overcomes the mobility issue of the trainees. The approach used in CORONA project ensured that the elaborated training schemes on VVER technology meet the International Atomic Energy Agency (IAEA) standards and the generally recognized criteria in the European Union (EU).

This project has received funding from the European Union Seventh Framework Programme (FP7-Fission-2011) under Grant Agreement No. 295999.

This material reflects only the author's view and the European Union is not liable for any use that may be made of the information contained herein.

\section{References}

[1] Nuclear Education and Training Key Elements of a Sustainable European Strategy, Sustainable Nuclear Energy Technology Platform Working group on Education, Training and Knowledge Management, December 2010, http://www. snetp.eu/www/snetp/images/stories Docs/ETKM/reportetkm.pdf

[2] Strategic Research Agenda, Sustainable Nuclear Energy Technology Platform, May 2009 http: // www.snetp.eu/www/snetp/images/stories/Docs-AboutSNETP/sra2009.pdf

[3] International Atomic Energy Agency, Nuclear Power Plant Personnel Training and its Evaluation A Guidebook, Technical Reports Series No. 380, IAEA, Vienna (1996)

[4] Von Estorff Ulrik, Corniani Enrico, Barboni Marcello, Karseka Tatiana, Multimedia Tool for WWER Training, NESTet 2013 - Transactions p. 273-280 